\title{
Study of Bound Nucleon Wave Functions by Coulomb Stripping in the Reaction ${ }^{208} \mathrm{~Pb}(\mathrm{~d}, \mathrm{p})^{209} \mathrm{~Pb}$
}

\author{
M. Dost and W. R. Hering \\ Max-Planck-Institut für Kernphysik, Heidelberg \\ (Z. Naturforschg. 21 a, 1015-1020 [1966] ; received 12 April 1966) \\ Dedicated to Professor Dr. W. Gentner on the occasion of his 60th birthday

\begin{abstract}
The differential cross sections of the ${ }^{208} \mathrm{~Pb}(\mathrm{~d}, \mathrm{p}){ }^{209} \mathrm{~Pb}$ transitions to the states $9 / 2^{+}, 5 / 2^{+}, 1 / 2^{+}$, $7 / 2^{+}$, and $3 / 2^{+}$in ${ }^{209} \mathrm{~Pb}$ were measured at $135^{\circ}$ laboratory angle for deuteron energies between 7.0 and 10.5 MeV. The data below $8.0 \mathrm{MeV}$ were analyzed in Coulomb Wave Born Approximation to give accurate spectroscopic factors of the five levels according to a simple shell model potential in the final nucleus. The dependence on some of the parameters that determine this potential is studied. The absolute value of the normalizing constants of the bound neutron wave functions outside the nuclear potential well are determined without reference to any shell model potential, i. e. their true values are given. It is confirmed that the ground and excited states up to $2.52 \mathrm{MeV}$ in ${ }^{209} \mathrm{~Pb}$ are pure single particle states so that from their study one can draw a proper shell model potential in the lead region.
\end{abstract}

Since many years the analysis of stripping reactions has been useful to gain spectroscopic information about nuclear states. Especially the DistortedWave-BonN-Approximation (DWBA) analyses give in general quite satisfactory results and so their basic validity is little doubted.

However, normally so many poorly determined parameters enter the DWBA that in some cases the extraction of spectroscopic factors ( $S$-values) as one of the most interesting pieces of physical information, becomes rather dubious.

The straightforward way out of this difficulty is the transition to low bombarding energies, i.e. Coulomb-stripping ${ }^{1}$ which reduces the number of free parameters for the DWBA since in that case the motion of the incoming and outgoing particles is independent of the nuclear potentials. Therefore the stripping cross section depends only on the bound nucleon wave function in the final nucleus. In particular one has:

1. If the bound state has a unique angular momentum $l$ its radial part outside the nuclear potential is given by a HANKEL function times a normalizing constant which can be measured directly.

2. In ideal cases where the $S$-values can be assumed to be known a priori, a parametrization of

1 L. C. Biedenharn, K. Boyer, and M. Goldstein, Phys. Rev. 104, 383 [1956]. - L. J. B. Goldfarb, Nucl. Phys. 72, 537 [1965].

2 R. H. Stokes, Phys. Rev. 121, 613 [1961].

3 J. R. Ersine, W. W. Buechner, and H. A. Enge, Phys. Rev. 128, 720 [1962]. the true shell model potential can be found for the mass region considered.

3. If an acceptable shell model potential for the single particle states in the final nucleus is known, one can measure with high accuracy absolute $S$ values of the states populated in stripping reactions.

Some experimental work on Coulomb stripping in heavy nuclei has already been done. Stokes ${ }^{2}$ has measured ${ }^{206,}{ }^{207} \mathrm{~Pb}(\mathrm{~d}, \mathrm{p})$ and ${ }^{209} \mathrm{Bi}(\mathrm{d}, \mathrm{p})$ at three bombarding energies. ERskine et al. ${ }^{3}$ have measured ${ }^{209} \mathrm{Bi}(\mathrm{d}, \mathrm{p})$ at two energies with high resolution. In both papers the authors concentrated their attention on the angular distribution in Coulomb stripping. The possibility of determining accurate $S$-values was studied in the reaction ${ }^{90} \mathrm{Zr}(\mathrm{d}, \mathrm{p})$ by BrIent et al. and by DaLLY ${ }^{4}$. These experiments finally gave the results expected if ${ }^{91} \mathrm{Zr}$ has a single neutron outside the $N=50$ shell.

To study the implications of Coulomb stripping in an experimentally and theoretically even cleaner case we measured the excitation functions of ${ }^{208} \mathrm{~Pb}(\mathrm{~d}, \mathrm{p})$ at $135^{\circ}$. It is the aim of this paper to extend the presentation of the results which in part have already been communicated in a short form ${ }^{5}$. So first in section I we will discuss the theoretical justification of our statements 1 . to 3 . In section II

${ }^{4}$ C. E. Brient, E. L. Hudspeth, E. M. Bernstein, and W. R. SMith, ORNL-TM-1102 [1965]. - E. DaLly, private communication.

5 M. Dost and W. R. Hering, Phys. Letters 19, 488 [1965]. 
the experimental technique is briefly described and the experimental results are given. Section III brings results of the DWBA-analysis. There we study the dependence of spectroscopic factors on the bound neutron potential and give the normalizing constants of the actual bound neutron wave functions in ${ }^{209} \mathrm{~Pb}$ and the parameters of an acceptable shell model potential in the lead region.

\section{Theoretical Remarks}

For a reaction $A(\mathrm{~d}, \mathrm{p}) B$ the differential cross section is in DWBA given by ${ }^{6}$ :

$\sigma=c|T|^{2} ; \quad T=\int \mathrm{d} \boldsymbol{r}_{\mathrm{p}} \mathrm{d} \boldsymbol{r}_{\mathrm{n}} \chi_{\mathrm{p}}^{(-) *}\left\langle B\left|V_{\mathrm{np}}\right| A\right\rangle \chi_{\mathrm{d}}^{(+)} \Phi_{\mathrm{d}}$

where $c$ is a statistical factor and the distorted waves $\chi_{\mathrm{p}}{ }^{(-)}, \chi_{\mathrm{d}}{ }^{(+)}$describe the relative center of mass motion of $(\mathrm{p}+B)$ and $(\mathrm{d}+A)$, respectively. The neutron-proton interaction is denoted by $V_{\mathrm{np}}$, $\Phi_{\mathrm{d}}$ represents the internal wave function of the deuteron. To avoid the sixfold integration $V_{\mathrm{np}}$ is almost always replaced by a constant $N_{d}$ times $\delta\left(r_{\mathrm{np}}\right)$ which means that $V_{\mathrm{np}}$ is assumed to have zero-range. Therefore all distorting effects of the Coulomb and nuclear fields on $\Phi_{\mathrm{d}}$ are neglected. This is certainly unjustified and has been subject to criticism ${ }^{7}$. However, it can be shown ${ }^{8}$ that in the normally used "post" formalism of DWBA one may use the zero-range approximation without making too serious a mistake.

The stripping form factor $\langle A \mid B\rangle \equiv \Phi_{\mathrm{n}}\left(\boldsymbol{r}_{\mathrm{n}}\right)$ can be expanded into a sum over shell model wave functions $\psi_{j l m}$. The problems of this expansion have been extensively discussed ${ }^{9}$. For our purpose it is important to note that outside the target nucleus, i. e. where the bound state potential has dropped off to zero we have

$\Phi_{\mathrm{n}}\left(\boldsymbol{r}_{\mathrm{n}}\right)=\sum_{l j m} S_{l j m}^{1 / 2} \psi_{l j m}=\sum_{l j m} S_{l j m}^{1 / 2} c_{l j} h_{l}^{(1)}\left(i k_{B} r_{\mathrm{n}}\right) Y_{l}^{m}\left(\hat{\boldsymbol{r}}_{\mathrm{n}}\right)$

where $\hbar k_{B}$ is the linear momentum that corresponds to the actual binding energy $E_{B}$ of the captured

6 N. K. Glendenning, Ann. Rev. Nucl. Sci. 13, 191 [1963]. G. R. SATCHLER, ANL-6878, 23 [1964].

7 S. T. Butler, Nature 207, 1346 [1965]. - L. J. B. GoldFARB and M. B. Hooper, Phys. Letters 19, 299 [1965].

8 F. B. Gibson and A. K. Kerman, On the Accuracy of Coulomb Stripping for Measuring Single Particle Reduced Widths in Nuclei, preprint, Oak Ridge 1966.

9 N. Austern, Phys. Rev. 136, B 1743 [1964]. - W. T. Pinkston and G. R. SAtchler, Nucl. Phys. 72, 641 [1965]. neutron. Since we are mainly interested in spin zero targets and heavier nuclei where seniority is assumed to be a good quantum number we may from now on restrict our consideration to cases of only one $l$-value in the expansion (2).

Then we have:

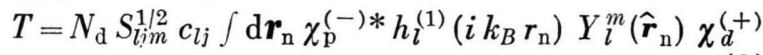

The functions $\chi_{\mathrm{p}}{ }^{(-)}$and $\chi_{\mathrm{d}}{ }^{(+)}$are calculated from optical potentials which have been determined by an elastic scattering analysis. Quite often this analysis does not give these potentials uniquely and if it does there may be serious objections against their use in stripping calculations ${ }^{10}$. The reason is that strong reaction channels may through virtual processes influence the elastic scattering but not the stripping. Therefore the optical potentials to be used may be not the same in both cases. However, if the scattering is determined by the Coulomb field alone, the distorted waves are known exactly and all further problems concern $N_{\mathrm{d}}$ and $S_{l j m}^{\frac{1}{2}} c_{l j}$ only. In this case we have Couloms stripping and it is clear that it will be possible only if the reaction $Q$-value is not too high and the bombarding energy is low. (In light nuclei we have the additional difficulty of compound nucleus effects which lead to fluctuations ${ }^{11}$.) To the extent that the structure of the deuteron is known from $n-p$ scattering the $N_{\mathrm{d}}$ itself may be considered as given. At low energies it is reasonable to take for $\Phi_{\mathrm{d}}$ a HuLthén wave function which gives $\left|N_{\mathrm{d}}\right|^{2}=1.5\left|N_{\mathrm{d}}^{0}\right|^{2}$, where $N_{\mathrm{d}}^{0}$ is the value for the zero-range deuteron wave function ${ }^{12}$. Actually this simple relationship between $N_{\mathrm{d}}$ and $N_{\mathrm{d}}^{0}$ is clear only for cases where the integrand in eq. (1) is a slowly varying function over the range of $V_{\mathrm{np}}$. At high incident energies where the interior part of $\Phi_{\mathrm{n}}$ with its comparatively rapid oscillations contributes the effect of using a HuLthéN wave function may be quite different. (The general aspects of using a finite-range wave function and nevertheless performing a zero-range calculation have been discussed by Buttre and Goldfarb ${ }^{13}$ and by Bencze and Zymanyi ${ }^{14}$.) In the case of

10 G. H. Rawitscher, Phys. Letters 21, 444 [1966].

11 L. L. Lee and J. P. Schiffer, Phys. Rev. 107, 1340 [1957].

12 R. H. Bassel, R. M. Drisko, and G. R. SAtchler, ORNL3240 [1962].

13 P. J. A. Buttle and L. J. B. Goldfarb, Proc. Phys. Soc. London 83, 701 [1964].

14 Gy. Bencze and J. Zimanyi, Phys. Letters 9, 246 [1964]. 
Coulomв stripping, however, the relevant part of $\Phi_{\mathrm{n}}$ is the monotonic Hankel function and the low energy protons and deuterons have long wavelengths. Therefore we regard $N_{\mathrm{d}}$ as fixed. So we are left with $S_{l j m}\left|c_{l j}\right|^{2}$ as the number which can be determined by Coulomв stripping, provided the $(l, j)$ of the final level is known. This is the model independent information we can gain, and we see from eq. (2) that this quantity is just the square of the coefficient of that part of the actual wave function $\Phi_{\mathrm{n}}$ which has angular momentum $l$ and therefore falls off as $h_{l}^{(1)}$.

Now the $c_{l j}$ actually are the normalizing constants of the shell model wave functions $\psi_{l j m}^{\bmod }$ used in their asymptotic forms in eq. (3) to calculate the theoretical cross sections and which we were free to choose. This means that for the experimental differential cross section one has $\sigma_{\mathrm{exp}}=S_{l j m}^{\bmod } \sigma_{\mathrm{DWBA}}^{\mathrm{mod}}$ where the model dependence of $S_{l j m}$ has its origin in the proportionality of $\sigma_{\mathrm{DWBA}}^{\mathrm{mod}}$ to $\mid c_{l j m}^{\bmod }{ }^{2}$, the square of the normalizing constant of $\psi_{l j m}^{\bmod }$. As soon as one knows the true $c_{l j}$ and therefore $\sigma_{\mathrm{DWBA}}$ one finds the true spectroscopic factor $S$ as the ratio $\sigma_{\exp } / \sigma_{\mathrm{DWBA}}$. This procedure implies that in reality there exists a true shell model potential which should be used.

On the other hand we can determine the true $\sigma_{\mathrm{DWBA}}$ and from this the $c_{l j}$ if we know the $S$-values a priori. This allows to find parameters that determine an acceptable shell model potential with eigenfunctions having the experimentally found normalizing constants outside the potential well. This is so especially if we have several states in a final nucleus where a single potential must give the expected $S$-values for all of them.

\section{Experimental Method and Results}

The $(l, j)$-values, excitation energies and $Q$-values of the transitions we studied, were taken from Mukherjee and Cohen ${ }^{15}$ and are given for con-

\begin{tabular}{|c|c|c|c|}
\hline $\begin{array}{c}\text { state } \\
\text { in }{ }^{209} \mathrm{~Pb}\end{array}$ & $\begin{array}{c}\text { excita- } \\
\text { tion } \\
\text { energy } \\
\text { MeV }\end{array}$ & $\begin{array}{c}\text { separa- } \\
\text { tion } \\
\text { energy } \\
\mathrm{MeV}\end{array}$ & $\begin{array}{c}Q \text {-value of reaction } \\
208 \mathrm{~Pb}(\mathrm{~d}, \mathrm{p})^{209} \mathrm{~Pb}(n l j)\end{array}$ \\
\hline $2 \mathrm{~g}_{9 / 2}$ & 0 & 3.94 & $\mathrm{MeV}$ \\
$3 \mathrm{~d}_{5 / 2}$ & 1.56 & 2.38 & 1.72 \\
$4 \mathrm{~s}_{1 / 2}$ & 2.03 & 1.91 & 0.16 \\
$2 \mathrm{~g}_{7 / 2}$ & 2.47 & 1.47 & -0.31 \\
$3 \mathrm{~d}_{3 / 2}$ & 2.52 & 1.42 & -0.75 \\
\hline
\end{tabular}

Table 1. Angular momenta, excitation energies, and $Q$-values of the five levels studied. The figures are taken from ref. ${ }^{15}$. venience in Table 1 . The measurements were carried out at the Heidelberg tandem VAN DE GraAfF with standard experimental techniques. We measured the ground state transition in a scattering chamber equipped with solid state detectors, which is described in the preceding paper by Richter et al. The Li-drifted silicon detectors were arranged at laboratory angles of $90,105,120,135,153$, and $171^{\circ}$. At room temperature they had an energy resolution for $12 \mathrm{MeV}$ protons of about $80 \mathrm{keV}$. The protons from transitions to excited states in ${ }^{209} \mathrm{~Pb}$ were detected on Ilford K2 and G5 photoplates in a broad range magnetic spectrograph which was described by Bock et al. ${ }^{16}$. Separation of deuterons by the magnetic field and by copper stopping foils was well possible without making the good energy resolution of about $20 \mathrm{keV}$ worse. Two typical spectra are shown in Fig. 1 which allows a comparison between the two detection methods.

The targets consisted of $99.9 \%$ enriched ${ }^{208} \mathrm{~Pb}$, $440 \mu \mathrm{g} / \mathrm{cm}^{2}$ thick at maximum, that was evaporated on a thin carbon film. To avoid a measurable evaporation of the lead during irradiation we limited the current to $250 \mathrm{nA}$ on the target at maximum. The target thickness was checked in regular intervals by taking the rate of elastically scattered deuterons. Thereby we checked also the inhomogeneity of the target to be less than $5 \%$.

In Table 2 are given the measured differential cross sections. We do not give values for the transitions to the $11 / 2^{+}$and $15 / 2^{-}$levels because their cross sections were too small according to the high

\begin{tabular}{|c|c|c|c|c|c|}
\hline $\begin{array}{c}\text { deuteron } \\
\text { energy } \\
\text { MeV }\end{array}$ & $2 \mathrm{~g}_{9 / 2}$ & $3 \mathrm{~d}_{5 / 2}$ & $4 \mathrm{~s}_{1 / 2}$ & $2 \mathrm{~g}_{7 / 2}$ & $3 \mathrm{~d}_{3 / 2}$ \\
\hline 10.50 & 1.36 & 5.45 & 4.12 & 1.81 & 5.61 \\
10.00 & 1.25 & 6.05 & 4.72 & - & 5.15 \\
9.85 & 1.14 & 5.33 & 3.75 & 1.41 & 4.55 \\
9.50 & 1.01 & 5.24 & 4.24 & 1.22 & 4.38 \\
9.00 & 0.70 & 4.22 & 3.55 & 0.81 & 3.37 \\
8.65 & 0.56 & 2.99 & 3.03 & 0.72 & 3.30 \\
8.50 & - & 2.94 & 2.19 & 0.40 & 2.31 \\
8.00 & 0.28 & 2.10 & 2.19 & 0.30 & 1.83 \\
7.50 & 0.13 & 1.41 & 1.57 & 0.17 & 0.98 \\
7.00 & 0.06 & 0.71 & 0.71 & - & 0.46 \\
\hline
\end{tabular}

Table 2. Differential cross sections (in $\mathrm{mb} / \mathrm{sr}$ ) for ${ }^{208} \mathrm{~Pb}(\mathrm{~d}, \mathrm{p}){ }^{209} \mathrm{~Pb}$ at $135^{\circ}$ laboratory angle.

15 P.Mukherjee and B.L. Cohen, Phys. Rev. 127, 1284 [1962]. 16 R. Bock, H. H. Duhm, S. Martin, R. Rüdel, and R. Stock, Nucl. Phys. 72, 273 [1965]. 


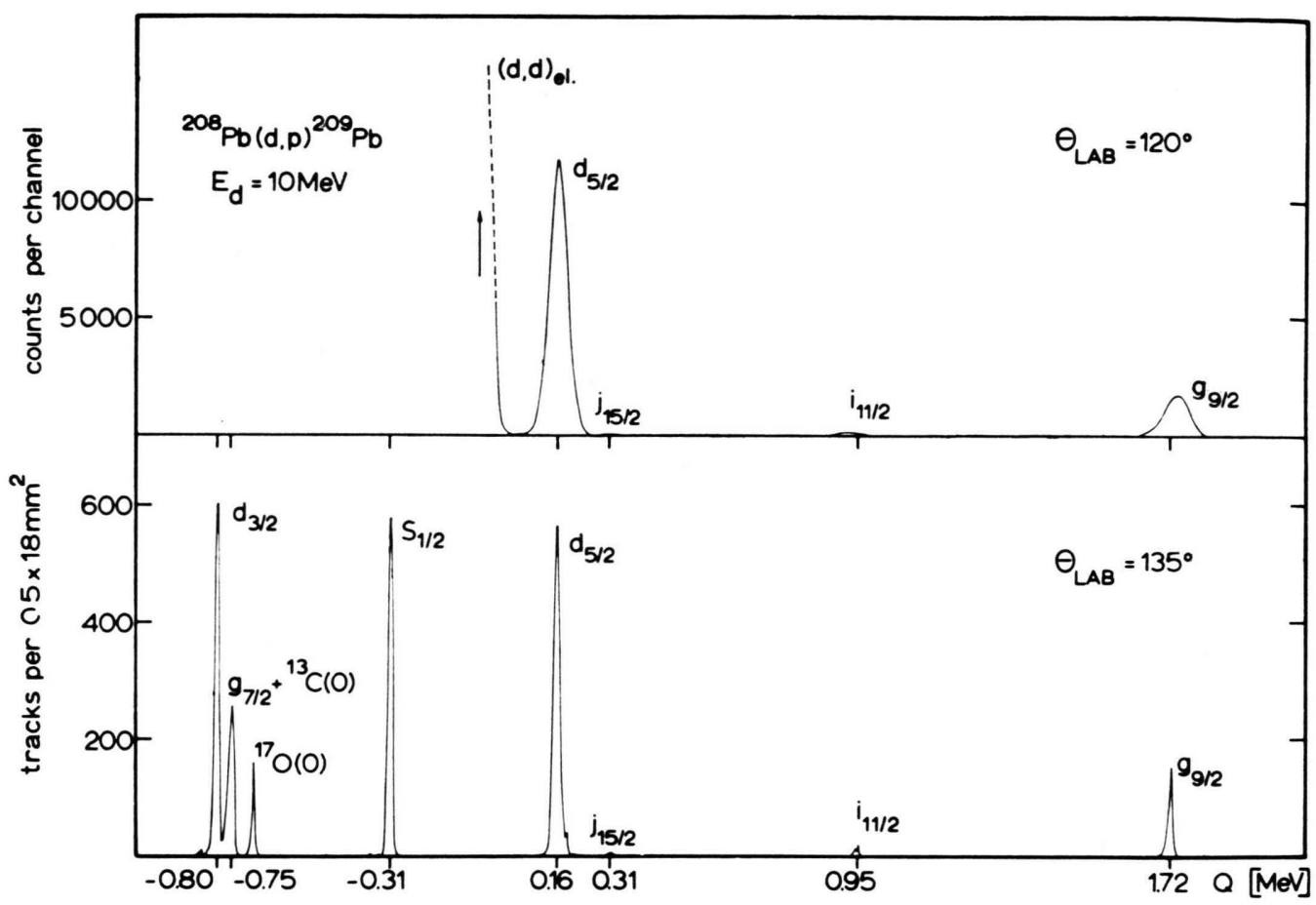

Fig. 1. Typical spectra taken in the scattering chamber (above) and the magnetic spectrograph (below). In the spectrograph the deuterons have been absorbed by copper foils.

orbital angular momentum transfer. A careful analysis of the experimental method led us to an estimate of the accuracy of $15 \%$ at every measured point.

\section{Discussion}

The stripping calculations were carried out with the DWBA code TSALLY of BASSEL et al. ${ }^{12}$, wherein the potential $V_{\text {pn }}$ is assumed to have zero-range. We compared at different bombarding energies the cross sections in Coulomb approximation with those in complete DWBA the scattering potentials of which were taken from Hodgson ${ }^{17}$. It was found that below $E_{\mathrm{d}}=8.0 \mathrm{MeV}$ the deviations between the two at $135^{\circ}$ were negligible or zero. Therefore we concluded that for ${ }^{208} \mathrm{~Pb}(\mathrm{~d}, \mathrm{p}){ }^{209} \mathrm{~Pb}$ and $135^{\circ}$ the Cолцомв stripping approximation is valid below 8.0 MeV. This is confirmed by increasing the optical potential depths by a factor of three which had almost no effect on the calculated cross sections below $8.0 \mathrm{MeV}$. On the other hand the DWBA curves with Hodgson's parameters show reasonable agreement with experiment also above $8.0 \mathrm{MeV}$.

\begin{tabular}{|c|c|c|}
\hline \multicolumn{3}{|c|}{$\begin{array}{l}\text { Analytical form of the scattering potentials: } \\
\begin{aligned} \text { deuteron: } & V_{\mathrm{c}}-(V+i W) \\
& \times\left[1+\exp \left\{\left(r-r_{0} A^{1 / 3}\right) / a\right\}\right]^{-1}\end{aligned} \\
\begin{aligned} \text { proton: } & V_{\mathrm{c}}-V\left[1+\exp \left\{\left(r-r_{0} A^{1 / 3}\right) / a\right\}\right]^{-1} \\
& -i W\left[\exp \left\{\left(r-r_{0}^{\prime} A^{1 / 3}\right) / a^{\prime}\right\}\right]^{-2}\end{aligned}\end{array}$} \\
\hline parameter & deuteron & proton \\
\hline $\begin{array}{l}V(\mathrm{MeV}) \\
W(\mathrm{MeV}) \\
r_{\mathrm{c}}(\mathrm{fm}) \\
r_{0}(\mathrm{fm}) \\
r_{0}^{\prime}(\mathrm{fm}) \\
a(\mathrm{fm}) \\
a^{\prime}(\mathrm{fm})\end{array}$ & $\begin{array}{l}50 \\
8.5 \\
1.5 \\
1.5 \\
- \\
0.65 \\
-\end{array}$ & $\begin{array}{c}58-0.3 \times E_{\mathrm{p}} \\
3 \times E_{\mathrm{pI}} 1 / 2 \\
1.25 \\
1.25 \\
1.25 \\
0.65 \\
0.98\end{array}$ \\
\hline
\end{tabular}

Table 3. Deuteron and proton scattering potentials taken from Hodgson ${ }^{17}$, used in the complete DWBA calculations. The Couloms potential is represented by $V_{\mathrm{c}}$, the potential of a homogeneously charged sphere of radius $r_{\mathrm{c}} A^{1 / 3}$.

Therefore they are given in Table 3 together with the analytical form of the scattering potentials.

As could be expected it turned out that the free parameters left for the description of the bound neutron entered the results quite strongly via the absolute value of the tail of the neutron wave func-

17 P. E. Hodgson, Proc. Padua Conference 1963, p. 103. 

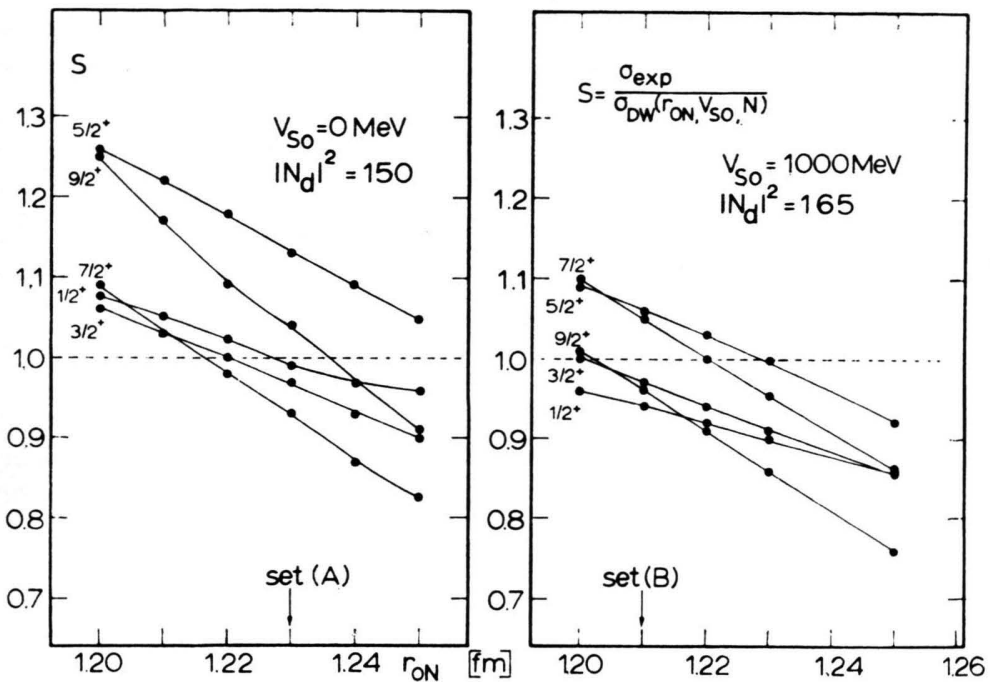

Fig. 2. Spectroscopic factors calculated with sets (A) and (B) and their dependence on the bound state potential radius $r=r_{0 \mathrm{n}} A^{1 / 3}$. tion. For the neutron potential we chose a WoodsSAxoN well of radius $r_{0 \mathrm{n}} A^{1 / 3}$, diffuseness $a$, and spin-orbit term of the form

$$
\begin{gathered}
-\left(\frac{\hbar}{2 M_{\mathrm{p}} c}\right)^{2} \lambda V(\boldsymbol{l} \cdot \boldsymbol{s}) \frac{1}{r} \frac{\mathrm{d}}{\mathrm{d} r}\left(e^{x}+1\right)^{-1} ; \\
\quad x=\left(r-r_{0 \mathrm{n}} A^{1 / 3}\right) / a .
\end{gathered}
$$

( $V$ is the depth of the Woods-Saxon potential which is fitted to the experimental separation energy $E_{B}$.) Two sets of parameters $\left(r_{0 \mathrm{n}}, a, \lambda,\left|N_{\mathrm{d}}\right|^{2}\right)$ turned

\begin{tabular}{|c|c|c|c|c|}
\hline & $r_{0 \mathrm{n}}$ & $a$ & $\lambda$ & $\left|N_{\mathrm{d}}\right|^{2}$ \\
\hline $\begin{array}{l}(A) \\
(B)\end{array}$ & $\begin{array}{l}1.23 \mathrm{fm} \\
1.21 \mathrm{fm}\end{array}$ & $\begin{array}{l}0.65 \mathrm{fm} \\
0.65 \mathrm{fm}\end{array}$ & $\begin{array}{r}0 \\
20\end{array}$ & $\begin{array}{l}1.5 \\
1.65\end{array}$ \\
\hline
\end{tabular}
out to give satisfactory results:

The corresponding spectroscopic factors are given in Table 4 . Since we could not carry out a systematic parameter search it is very likely that these two sets do not give the best description of the

\begin{tabular}{|c|c|c|c|}
\hline $\begin{array}{c}n l j \text { of state } \\
\text { in } 209 \mathrm{~Pb}\end{array}$ & $\begin{array}{c}S_{l j m} \\
\text { set (A) }\end{array}$ & $\begin{array}{c}S_{l j m} \\
\text { set (B) }\end{array}$ & error \\
\hline $2 \mathrm{~g}_{9 / 2}$ & 1.07 & 0.96 & $\pm 14 \%$ \\
$3 \mathrm{~d}_{5 / 2}$ & 1.14 & 1.06 & $\pm 10 \%$ \\
$4 \mathrm{~s}_{1 / 2}$ & 1.11 & 0.94 & $\pm 9 \%$ \\
$2 \mathrm{~g}_{7 / 2}$ & 0.98 & 1.05 & $\pm 15 \%$ \\
$3 \mathrm{~d}_{3 / 2}$ & 1.01 & 0.97 & $\pm 11 \%$ \\
\hline
\end{tabular}

Table 4. Spectroscopic factors $S_{l j m}$ for the two bound state potentials (A) and (B) given in the text. asymptotic neutron wave functions. But one can see that the inclusion of spin-orbit coupling reduces the mean deviation of the $S$ from 1 considerably, making it $6 \%$ at maximum.

The different value for $\left|N_{\mathrm{d}}\right|^{2}$ in potential $(B)$ comes from a change in the Hulthén function due to recent $n-p$ scattering analyses ${ }^{18}$. Since we changed only $r_{0 \mathrm{n}}$ it might be equally interesting to know how the diffuseness $a$ influences the $S$-values. This is shown in Table 5 for $l=0$ and $l=4$. Obviously there is no reason to prefer the variation of $r_{0 \mathrm{n}}$ to that of $a$, and in a systematic parameter search both will have to be varied.

\begin{tabular}{|c|c|c|c|c|c|c|}
\hline $\begin{array}{c}n l j \text { of } \\
\text { state in } \\
209 \mathrm{~Pb}\end{array}$ & 0.55 & 0.60 & 0.65 & 0.70 & 0.75 & 0.80 \\
\hline $2 \mathrm{~g}_{9 / 2}$ & 1.21 & 1.08 & 0.96 & 0.85 & 0.76 & 0.67 \\
$4 \mathrm{~s}_{1 / 2}$ & 1.12 & 1.03 & 0.94 & 0.86 & 0.79 & 0.73 \\
\hline
\end{tabular}

Table 5. Variation of the spectroscopic factors $S_{l j m}$ from set (B) with diffuseness $a$ in the $l=0$ and $l=4$ cases.

Finally it is one of the main results of our experiment that the possibility of fitting all five transitions by one parameter set within experimental errors to $S_{l j m}=1$ supports the expected single particle nature of the five states studied in ${ }^{209} \mathrm{~Pb}$. In this sense the numbers given in Table 6 are the normalization constants $\left|c_{l j}\right|$ of the actual bound neutron wave functions.

18 G. R. SAtchleR, private communication. 


\begin{tabular}{|c|c|c|c|c|c|}
\hline $\begin{array}{c}n l j \\
\text { of state } \\
\text { in }{ }^{209} \mathrm{~Pb}\end{array}$ & $2 \mathrm{~g}_{9 / 2}$ & $3 \mathrm{~d}_{5 / 2}$ & $4 \mathrm{~s}_{1 / 2}$ & $2 \mathrm{~g}_{7 / 2}$ & $3 \mathrm{~d}_{3 / 2}$ \\
\hline$\left|c_{l j}\right|$ & 0.69 & 1.26 & 2.09 & 0.037 & 0.39 \\
\hline
\end{tabular}

Table 6. Absolute values of the coefficients $c l j$ in the Hankel function expansion of the actual from factor $\Phi_{n}\left(\boldsymbol{r}_{n}\right)$.

In conclusion we may say that potential $(B)$ is quite a good approximation to the shell model potential in the lead region. (In particular, the $\left|N_{\mathrm{d}}\right|^{2}=1.65$ seems to be better than 1.5 for the Hulthén wave function.) With this potential one can hope to find reasonably accurate spectroscopic factors in all cases

19 H. Meldner and G. Süssmann, Phys. Letters 6, 353 [1963]. - H. Meldner, G. Süssmann, and W. Ulrici, Z. Naturforschg. 20 a, 1217 [1965]. where one $l$-component of an eventual mixture dominates. What changes are caused by the nonlocality of the potential ${ }^{\mathbf{1 9}}$ is not yet sufficiently studied. They may be large and in any case will have to be included in a best possible approximation of the true shell model poential. Our experimental data seems to be accurate enough to allow the tackling of such a problem.

We would like to thank Dr. R. Bock and his group for their kind help during the measurements with the Buechner magnet. We also thank Drs. G. H. Rawitscher, K. Dietrich, C. Noack, and W. R. Smith for numerous clarifying discussions. 